\title{
A Cross-Cultural Study on the Cognitive Traits That Affect Hindsight Bias —Focusing on Holistic-Analytic Thinking and Conservatism
}

\author{
Han W. Jung \\ Incheon, Republic of Korea ${ }^{1}$
}

\begin{abstract}
This study is an attempt to replicate a certain theory about the East-West cultural differences in cognitive tendencies. Nisbett (2003) theorized that there are cultural differences in cognitive styles between Eastern and Western societies, with Eastern societies tending towards holistic thinking and connecting objects through relationships and Western societies tending towards analytic thinking and sorting objects through categorization. Whereas Westerners tend to see the essence of nature itself, Easterners tend to focus on the harmony and interaction between humans and objects. As an extension of this claim, Choi and Nisbett (2000) indicate that Koreans have stronger hindsight bias than Westerners. However, this study successfully invalidated their studies, demonstrating that there was little East-West difference in the way of thinking and Koreans' strong hindsight bias resulted from their higher conservatism compared to Westerners. The results found no significant difference in hindsight bias between Koreans and Westerners. In addition, there was no East-West difference in categorization-relationship and essence-interaction preferences, although conservatism was found to be higher in Koreans than Westerners. The multiple regression model showed that the differences in such preferences did not affect hindsight bias and conservatism was the only factor that increased hindsight bias.
\end{abstract}

\footnotetext{
${ }^{1}$ Now I am a master's student at Illinois State University. For comments or concerns, please contact hjung15@ilstu.edu.
} 


\section{Comment from the Future}

This study was conducted and delivered in 2014 to Dr. Incheol Choi, the person directly involved in this study, but I could receive no response from him at that time. Six years later, I decided to release this study because I hoped that my research might bring a little help with developing in a desirable direction of the contemporary cultural psychology and sounding an alarm about the current manner of cross-cultural studies, as well as I realized that not releasing the completed study could also be a file-drawer bias.

The dichotomy between the East-West cultures, prevalent in the current cultural psychology field, does not seem to have been corrected in the past six years but rather seems to have spread to laypeople as well, playing a role in aggravating certain forms of cultural prejudice including 'orientalism' that considers Eastern cultures as absolute outgroups and tries to explain every Eastern social phenomenon with the collectivist Confucian culture. I thought it is time that a special caveat is needed and even a clumsy voice from a novice researcher would be enough to help someone a little.

This study was performed without human subject review or IRB approval. In the survey, the contents of the study and the information collected were briefly notified at the top of the questionnaire, and the participants were considered to be agreeing to participate in the study when they start to answer the questions. As this study was conducted in 2014, it follows the APA 6th guideline. 
Hindsight bias is the phenomenon in which we start to think the certain events had been predictable after they actually happened. People are inclined to say as if they have known it all beforehand even though in reality, they did not know it would happen before the event. Since its first scientific discovery by Fischhoff (Fischhoff, 2007), hindsight bias has been one of the hottest issues in cognitive psychology for more than 40 years. Among them, theories of the cross-cultural differences in hindsight bias, established by Incheol Choi and Richard E. Nisbett, caught my attention. They argued that Korean students tend not to be surprised at the unexpected results compared to Western students, thinking that the results were predictable, which indicates that Koreans generally have more hindsight bias than Westerners (Choi \& Nisbett, 2000).

They explained that this phenomenon stemmed from Koreans' higher preference for holistic thinking. Nisbett has argued that the cultural differences between East and West societies not only exist in tangible elements but also in people's cognitive styles: Asians prefer holistic thinking that focuses on comprehensiveness, relationship, and harmony, whereas Westerners prefer analytic thinking that focuses on individual, essential, and logical values (Nisbett, 2003, Nisbett, Peng, Choi, \& Norenzayan, 2001). According to this perspective, Western culture has been affected by Greek philosophy and the logical way of thinking and reasoning so that it has strong individualism and independent outlook, focusing on the objects themselves. In contrast, Easterners have been influenced by Chinese culture that has emphasized the relationships among people; Thus, they are mostly collectivists who are interdependent and focus on backgrounds or contexts.

However, some questions remain. First, the differences in hindsight bias shown in their studies seem too big to result from a single variable. In my opinion, only two reasons can explain this distinct difference: The unexpected noises appeared in the experiment process or the different factors besides holistic thinking that may increase hindsight bias. Second, several studies are indicating the different results from theirs. Pohl, Bender, and Lachmann (2002) suggested that the Easterners' hindsight bias is not higher than the Westerners'. Heine and Lehman (1996) proposed the result that Japanese' hindsight bias is slightly lower than the Westerners, which is unlikely to happen if the holistic view actually initiates hindsight bias. Third, it is still unknown by what mechanisms holistic thinking causes greater hindsight bias. Unlike their confident assertions, their model fails to show any satisfactory models or mechanisms that can specify the causal relationship: Indeed, this theory is usually based on anecdotal clues rather than empirical verification, which are too vague to establish perspicuous evidences to demonstrate the impact of holistic thinking on such differences.

Hence, first I hoped to check whether Koreans' hindsight bias is really higher than Westerners and if so, whether there are some other reasons that can explain why Koreans have higher hindsight bias. I assumed that for the first statement to be true, there should be certain kinds of individual differences in hindsight bias and such differences should be connected to the cultural attributes that distinguishes between Koreans and Westerners. At least, it is true that there are some individual differences in hindsight bias. According to Musch (2003), hindsight bias is related to the following characteristics: social desirability, impression management, dogmatism, rigidity, field dependence, and conscientiousness. Among these characteristics, I primarily concentrated on the dogmatism, rigidity, and conscientiousness because these are also related to conservative personality (Gerber, Huber, 
Doherty, Dowling, \& Ha, 2010, Jost, Glaser, Kruglanski, \& Sulloway, 2003). Although these dispositions have been treated as individual factors in previous research, it is possible their shared relationships with hindsight bias may be derived from the single factor: conservatism. My inference was that Koreans are averagely conservative than foreigners; Positing that, Koreans' strong hindsight bias would result from the conservative cognition.

The second question was whether Koreans are truly more holistic than Westerners. Although Choi, Koo, and Choi (2007) conducted the experiment that ostensibly prove Koreans' higher holistic way of thinking compared to Westerners or Americans, the situation becomes more suspicious when more closely looking at this study. This study adopted the seven-point Likert scales to compare the five factors related to holistic thinking, but Koreans' average (4.99 5.48) was not much bigger than Americans' (4.68 5.06). In my opinion, it is more important that they all preferred holistic thinking to analytic thinking. The average of $4.5 \sim 5$ means the strong agreement in the seven-point Likert scale. Is it meaningful to compare the group difference if almost all participants agree with the questions?

Furthermore, the claim that Koreans or Asians are more holistic than Westerners does not seem to have a sufficient theoretical rationale. In his book The Geography of Thought, Nisbett presented the following experiments to show the difference in mindset between Oriental and Western culture (Nisbett, 2003). Participants looked at pictures of three objects (e.g., monkey, panda, and banana) and were asked to select pairs that match well. In this situation, there are two ways to answer. First, one could categorize the objects, connecting objects that share the same attributes (e.g., pairing monkey and panda, as both are mammals). Second, one could pair objects by their relationships (e.g., connecting monkey with banana because monkeys eat bananas). Nisbett argues that Westerners tend to pair monkey and panda, whereas Asians pair monkey and banana, indicating a Western preference for categorization and an Eastern preference for sorting by relationships. Once again, is it unreasonable to think that only the cultural background made their choice so distinct like this? I supposed that even though the results were numerically true, his interpretation that this difference was all derived from the cultural differences in cognitive tendency is too hasty and does not have enough theoretical grounds. At least, the monkeypanda-banana question is not an appropriate item to measure holistic thinking because the cultural backgrounds or circumstances which are unrelated to holistic thinking may impact the strength of the association between monkey and banana. I speculated that because monkeys have been historically familiar or even sacred animals in Asia whereas Europe and North America are not the natural habitats of monkeys, most Westerners may not have the image of monkeys as sturdy as the Oriental people. Therefore, they cannot easily imagine monkeys eating bananas as Asians and just classify them through the categorization which is conceivable in the next step.

For this reason, I wanted to see the experiments with other items besides the monkeypanda-banana. In the study of Ji, Zhang, and Nisbett (2004), the same type of word group experiment mentioned above was conducted to the three groups of participants: Chinese, Chinese living in the US, and Americans, respectively. They also added a device to find out the impact of language on the answers: Chinese and Chinese in the US were divided into two groups and asked to answer the questions in Chinese and English, respectively. The result indicated that the differences existed not only between-groups but also within Chinese in the US groups according to the language they used. However, I had to doubt the 
objectivity of this study because they seem to have made biased decisions when selecting the word groups. The most critical problem was the following groups: postman-policemanuniform and teacher-doctor-homework. In English, it is easy to choose the pairs of postmanpoliceman and teacher-doctor because they have the same or similar suffixes, but in Chinese, they are entirely different word\$2 ${ }^{2}$. Therefore, we cannot say that the cultural background or language affected their way of thinking, but the difference seems to have come from the researchers' biased word choice.

Another point to mention was the fundamental attribution error (FAE) because Choi and Nisbett (1998) argued that Asians, who prefer holistic thinking, are less susceptible to the fundamental attribution error compared to Westerners who prefer analytic thinking. In this study, the participants, who were either Koreans or Americans, were asked to read texts that support or oppose the death penalty and estimate the writers' actual stances on the death penalty. In this situation, the participants were divided into two groups: The first group was told that the writers could choose their position before writing, but the second was told that they could not choose the position. For their hypothesis to be correct, the difference in the first and second group must have been greater in Americans than Koreans. However, the results showed that both Koreans and Americans had strong FAEs, and the difference was even higher in Koreans than Americans. They concluded that this unexpected result was due to the lack of situational constraints and decided to carry out the second experiment. They added some situational constraints in the second experiment, then the FAE in Koreans decreased more than one in Americans.

Nevertheless, they used this result only to reinforce their existing claims. In his book The Geography of Thought, Nisbett did not mention the first experiment of this study but only the second to insist that Koreans have lower FAEs than Americans. Choi, who had translated this book into Korean, also adhered to this argument in his later articles and books. Other studies on cross-cultural differences in the FAE do not support their claims, as well as their first experiment. Krull et al. (1999) showed that there was no difference in FAE between individualist and collectivist cultures. Gong (1996) argued that the Koreans' tendency of attribution is analogous to the Americans.

Nisbett's deficient argument and reasoning are also in his book itself: His book contains lots of logical contradictions that made me doubt that he is a Westerner. For example, after he cited the article on page 84 which represented that the cultural difference in the cognitive style appeared in two-year-old children, he started to claim on page 195 that the East-West cultural differences originated in the metaphysical beliefs. Furthermore, arguing that Asians have lower FAEs, he also presented a study on page 71 which reported that the group stimulated by collectivist thinking was more likely to criticize others.

Those I have discussed so far were mostly known during the study. At first, I started this study from the small question about the argument of two eminent social psychologists. However, it did not take much time that the little question snowballed into the horrifying conviction.

\footnotetext{
${ }^{2}$ postman (邮递员tóudìyuán)-policeman (警察jǐngchá), teacher (教师jiàoshī)-doctor (医生yīshēng, 医 师yīshī). I could not find which word this study used, but generally, 医生yīishēng is the common word that means doctor.
} 


\section{Methods}

\section{What to Measure}

To see whether Koreans really prefer holistic thinking more than foreigners, first of all, we have to determine how to measure holistic thinking. There was a measurement that Choi et al. (2007) developed, but I thought that it had too many items, low reliability, and limitations in measuring a wide range of holistic thinking. There was also the problem of acquiescence: The response was concentrated only on the side of agreement.

In this study, I used two separate measurements. First, the participants were given three words such as the monkey-panda-banana experiment mentioned above and asked to choose two of them. I decided to maintain the format of the Nisbett's experiment, but I tried to remove all the language biases in selecting the words to make sure that all unintentional noises to be removed. Second, certain types of example sentences conceptually considered as Nisbett's holistic and analytic thinking were shown to the participants, and they were asked to choose which one was closer to their way of thinking. As mentioned above, holistic thinking emphasizes relationship and harmony whereas analytic thinking pursues essence, rationality, and unchanging truth.

Would the difference between Koreans and foreigners exist in these scales? I did not think so. There might be slight differences, but it would not be as huge as Nisbett argues. I presumed that most studies supporting the 'big difference' in cognitive style between cultures are just a statistical artifact contaminated by noises. But on the other hand, I supposed that the East-West cognitive differences exist in different ways: cognitive conservatism, which means that Koreans are more likely to be cognitively conservative than Westerners. The conservatism here refers to the internal cognitive characteristics of preferring stability and status quo, pursuing traditional and patriarchal values, and showing resistance and fear of sudden changes. I believed that this tendency would be stronger in Koreans than in foreigners, and further, this would be a significant factor to explain the differences that the existing concept of cultural psychology cannot explain.

In the case of hindsight bias, I supposed that it would be larger in Koreans as Choi's argument; Choi and Nisbett argued that Asians are mostly 'field dependent' compared to Western people, which means that Asians are more likely to be affected by contexts or backgrounds, and it is known that the field dependence has a strong correlation with hindsight bias (Musch, 2003). However, I took a different position from Choi on why Koreans have greater hindsight bias. If I was to consider only two factors, field dependence and conservatism, the primary origin of hindsight bias would be conservatism because I assumed above that Koreans are mot more holistic than Westerners. Nevertheless, I thought that the existing theory that people who prefer holistic thinking have greater hindsight bias is true because the field dependence of holistic people can increase hindsight bias.

Then why does the conservative orientation raise hindsight bias? The simplest explanation is that it is due to conservative people's dogmatism, rigidity, and conscientiousness, as mentioned above. However, I was more concerned about the model that allows the methodical explanations of people's cognitive process. For this reason, I decided to utilize Pezzo (2003)'s sense-making model: He argued that when people encounter the unexpected truths and are surprised, they tend to find plausible reasons to rationalize it (sense-making), and if the process of making sense is successful, then their hindsight bias increases, but if 
the process fails, it decreases instead.

In this regard, I thought that conservative people tend to be easier to make sense and such tendency will consequentially raise hindsight bias. My conjecture was that such phenomenon results from the cognitive difference between the conservatives and nonconservatives; As the conservatives have lower integrative complexity of thinking (Tetlock, 1983 ) and report weaker volume of anterior cingulate cortex, which monitors the errors of information (Kanai, Feilden, Firth, \& Rees, 2011), they tend readily to believe the specious information. In contrast, the non-conservatives are likely to realize the flaws in their previous sense-making, leading to the drop in hindsight bias. To identify this cognitive difference, I decided to make a scale that measures how readily people can be confident of their thought and find out its relationship with conservatism and hindsight bias. This scale was named 'negligence' and aims to measure the propensity to make sense in a short time rather than to think deeply and cautiously.

\section{Measures, Design, and Organization}

The survey was conducted among the Korean and foreign participants recruited on the streets of the Seoul Metropolitan Area in the Republic of Korea. The questionnaires were produced in Korean and English at the same time and translated solely by myself. All surveys were conducted anonymously, and I did not collect any demographic information except the age, gender, and occupation. However, the foreigners' nationality and length of residence in Korea were additionally asked.

The monkey-panda-banana scale consists of a total of six items, each of which presents three words and asks the participants to choose two of them. I designed each item to make sure that among the three kinds of answers, one response based on the categorization and another based on the relationship are possibl ${ }^{3}$. The response based on the categorization is worth zero points and the response based on the relationship is worth two points, so the possible score range of this scale is $0-12$. If the participants made the third response which is not based on either the categorization or relationship, it is worth one point, and this happened 18 times out of a total of 1,440 trials. The reliability of this scale was estimated through the split-half method which calculates the Spearman-Brown correlation between the average of the odd-numbered items and the average of the even-numbered items.

The essence-interaction-way-of-thinking (WoT) scale consists of four items. Each item presented two descriptions regarding the same issue, one of which was an essentialistic (related to analytic thinking) description and the other interactive (related to holistic thinking). Then the participants were to answer which description is closer to their way of thinking. The questions were made with the four-point scales. A preference for essence (A) is worth zero points. A slight preference for essence is worth one point, and a slight preference for interaction (B) is two points. A preference for interaction is worth three points, and the score of each participant is the sum of four questions which ranges from 0 to 12 .

Conservatism was measured with five items asking participants whether they agree or disagree (seven-point Likert) with statements regarding conservative attitudes or beliefs.

\footnotetext{
${ }^{3}$ Please note that the actual 'monkey-panda-banana' word group was not included in this study because I thought that this word group is inappropriate to measure the preference for the categorization or relationship suitably.
} 
There were no reverse-scored items, and in all items, the more the participants agree, the more conservative they are. Therefore, people who agree with the sentences would get the higher score, which means that they are more conservative. The contents of the items consist of the conservative outlook, traditionalism, patriarchal ideology, resistance to change, and antipathy towards liberalism. As I intended to measure the cognitive aspects of conservatism, I removed all elements that are explicitly political or might be affected by specific political or social backgrounds. The Cronbach's $\alpha$ coefficient estimated the reliability of this scale.

Furthermore, I confirmed the external validity of the conservatism scale through the correlation between the measured conservatism of the participants and their real political tendencies. For this, I directly asked some participants' political stances: I asked Koreans which candidate they voted in the 18th presidential election and asked foreigners which American party they supported (or preferred if they are not Americans) between the Democratic and Republican parties. For the conservatism scale to be valid, their political choices should be split up according to the conservatism scale. Besides, the participants' self-reported political stances were also asked. This question consists of the five-point Likert scale from liberal to conservative, and the correlation between their self-reported political stances and the measured conservatism was also used to check the validity of the conservatism scale.

Hindsight bias was measured with each participant's difference in the hindsight opinion (credibility) of two conflicting arguments. The participants were asked to see two opposing and seemingly contradictory arguments (A and B) and told that one of the two arguments is correct and the other is wrong. They were divided into two groups, and one group was told that argument A is correct whereas the other group was told that argument $\mathrm{B}$ is correct. Then on the next page, they were asked to indicate as the seven-point Likert scales how much they thought that arguments A and B were correct on the previous page, respectively ${ }^{4}$

Although it looks that the two arguments are fighting each other, they were actually both fabricated theories. They both have the style of reasoning that combines the trumped-up research findings with plausible reasons to derive absurd conclusions or at least conclusions without any evidence. Argument A said that people in economically powerful countries have higher moral standards, and argument B said that the national wealth is inversely proportional to the people's morality. But in fact, there is no scientific evidence showing that the national economic power correlates with people's morality.

By the way, this measurement needs the control group that confirms people's foresight credibility, which means how much they would think arguments A and B were correct if they were not told which of the two arguments was correct. For this, I asked some participants to answer how much they believed the arguments were correct without notifying which one was correct. At this point, I intended to find out whether the foresight credibility of ordinary people was all the same. If the credibility of one argument is disproportionately high, the difference between arguments A and B cannot reflect the participants' hindsight change of selection, and the measurement error will increase as a result. However, if the foresight credibility of A and B is revealed to be the same in all people as my assumption,

\footnotetext{
${ }^{4}$ In the actual questionnaire, there were two more questions asking how much surprising and interesting the presented fact was. These questions were used subsidiarily in the analysis.
} 
I can use the formula of difference as follows to measure hindsight bias:

$$
h b_{a}=h_{a}-h_{b}, h b_{b}=h_{b}-h_{a}
$$

In the formula above, $h b_{m}$ indicates hindsight bias of when argument $\mathrm{M}$ is given to be correct, and $h_{n}$ indicates hindsight credibility of argument N. As I will measure hindsight bias through the difference between the experimental group member's hindsight credibility and the control group member's foresight credibility, the hindsight bias in this study will be equal to (an experimental group participant's credibility of argument A) - (a control group participant's credibility of argument A) - (an experimental group participant's credibility of argument B) + (a control group participant's credibility of argument B). However, assuming that the foresight credibility of arguments A and B are all the same, they are offset by each other and disappear, and hindsight bias can be measured only with the data of the participants in the experimental group.

This manipulation is crucial for this study because it enables to measure individual differences in hindsight bias and perform a regression analysis beyond the mere comparison of differences between groups. According to this formula, hindsight bias was measured as a continuous variable from -6 to 6 , and I posited that lower scores mean lower hindsight bias even if they were negative values. The reliability of this scale was measured by the Spearman-Brown correlation between the credibility of argument A and the reverse-scored credibility of argument B.

Lastly, I measured the negligence with two item:5. These items are a seven-point Likert scale and measured their self-reported overconfidence in their beliefs, judgments, or intuitive decisions. The Cronbach's $\alpha$ estimated the reliability of this scale.

Piecing together the contents designed so far, I divided the questionnaire into three types. When measuring hindsight bias, type A presents the description that argument $\mathrm{A}$ is correct and B is wrong. Type B presents the opposite. Type C measures the control group's foresight credibility. All types of questionnaire include the monkey-panda-banana, the WoT, and the conservatism scales and only type $\mathrm{C}$ measures the validity of the conservatism scale. The negligence scale was only included in types A and B because it was used only to measure the relationship between conservatism and hindsight bias.

Therefore, the questionnaire types A and B consist of monkey-panda-banana, the WoT, hindsight bias, conservatism, and negligence scales, and type C consists of monkeypanda-banana, the WoT, the control group of hindsight bias, conservatism, and the items that measure the validity of the conservatism scale. Types A and B differ only in the description of which argument is correct and which is wrong in the hindsight bias scale. All three types had the same sample count of 80 people (40 Koreans and 40 foreigners) and was distributed randomly to the respondents under the same conditions. The contents of the questionnaire are summarized in Table 1.

The hypotheses of this study are as follows: First, Koreans will have higher hindsight bias than foreigners. Second, there will be no significant differences between Koreans and foreigners in the monkey-panda-banana scale. Third, Koreans' conservatism will be higher

\footnotetext{
${ }^{5}$ At the beginning, it was four items including one reverse-scored item, but I analyzed only two of them due to the problem of reliability and validity.
} 
Table 1

Summary of the items used in the study.

\begin{tabular}{cccc}
\hline Scale & Type & No. & Item \\
\hline & & $1-1$ & cow, horse, saddle \\
Monkey- & & $1-2$ & honey, bee, sugar \\
panda- & A $+\mathrm{B}+\mathrm{C}$ & $1-3$ & pencil, graphite, ballpoint \\
banana & & $1-4$ & burger, water, coke \\
& & $1-5$ & racket, tennis ball, golf ball \\
& & $1-6$ & train, airplane, wing \\
\hline Essence- & & $2-1$ & Organization \\
interaction & \multirow{2}{*}{$\mathrm{A}+\mathrm{B}+\mathrm{C}$} & $2-2$ & Literature \\
way of & & $2-3$ & Nature \\
thinking (WoT) & & $2-4$ & Science \\
\hline \multirow{2}{*}{ Conservatism } & & $4-1$ & General description of conservative identity \\
& & $4-2$ & Conformity to traditional values \\
& & $4-3$ & Discriminatory attitude against women \\
& & $4-4$ & Resistance to social change \\
Hindsight bias & \multirow{2}{*}{$\mathrm{A}+\mathrm{B}$} & $3-3$ & How much one thought argument A was true \\
& & $3-4$ & How much one thought argument B was true \\
\hline \multirow{2}{*}{ Negligence } & \multirow{2}{*}{$\mathrm{A}+\mathrm{B}$} & $4-6$ & Overconfidence in one's thoughts \\
& & $4-7$ & Overconfidence in one's intuitive judgments \\
\hline
\end{tabular}

than foreigners'. Fourth, the monkey-panda-banana scale, the WoT scale, and the conservatism scale will all be correlated with greater hindsight bias. Fifth, conservatism will increase negligence, and negligence will increase hindsight bias again so that the negligence will mediate the relationship between conservatism and hindsight bias. 


\section{Results and Discussion}

\section{Pre-Analysis}

Demographics. The participants were 120 Koreans and 120 foreigners. Only foreigners who entirely seemed to be Westerners were surveyed, and those who looked like Asians or mixed Asians were excluded. The survey started on May 3, 2014 and ended on August 14, 2014. The statistical analyses were performed with SPSS, and some functions not provided by SPSS were performed with external calculators. The p-values calculated in this study are all based on the two-tailed test.

The foreigners were surveyed in Insa-dong (62.5\%) and Itaewon (37.5\%), and Koreans were surveyed in Insa-dong (26.7\%), Beomgye-dong (59.2\%), and Yongsan station (14.1\%). The average age of Koreans was $27.8(S D=8.33)$, and the average of foreigners was 29.6 $(S D=8.35)$. Among the Korean participants, $71(59.2 \%)$ were male and $49(40.8 \%)$ were female, whereas for foreigners, $72(60 \%)$ were male and $48(40 \%)$ were female.

Of the 120 foreigners, 58 (48.3\%) were Canadians or Americans; 40 (33.3\%) were Western/Central/Northern Europeans or Oceanians; 17 (14.2\%) were Southern/Eastern Europeans or Latin Americans; 5 (4.2\%) were Africans, Arabians, or Asians. Each national identity was considered as each participant's nationality type, and the participants were categorized into a total of five nationality types, including the type of Koreans. About a half $(62,51.7 \%)$ of foreigners had stayed in Korea for less than two months, and the rest $(58,48.3 \%)$ had lived in Korea for two months or more. The average length of residence of foreigners was 9.9 months $(S D=18.13)$.

Reliability. For the monkey-panda-banana scale, the correlation between the average of the odd-numbered items and one of the even-numbered items in the questionnaire type $\mathrm{A}+\mathrm{B}+\mathrm{C}$ was .553 , and the reliability estimated by the Spearman-Brown formula was .712. The estimated reliability was a little higher in Koreans (.800) than in foreigners (.614).

For the WoT scale, the Cronbach's $\alpha$ in type A+B+C was .227. The coefficient of Koreans was .219, and one of the foreigners was .267, indicating that both Koreans and foreigners did not answer the questions consistently.

The Cronbach's $\alpha$ of the conservatism scale in type A+B+C was .793. The Koreans' coefficient was .732, and the foreigners' was .827. The Cronbach's $\alpha$ of the negligence scale in type $\mathrm{A}+\mathrm{B}$ was .651 in Koreans, .691 in foreigners, and .663 in all participants.

For the hindsight bias scale, the correlation between the hindsight credibility of argument $\mathrm{A}$ and one of argument $\mathrm{B}$ was -.485 in type $\mathrm{A}+\mathrm{B}$, and the reliability estimated by the Spearman-Brown formula was .653. The estimated reliability was higher among the foreigners: The reliability in Koreans was .533, and one in the foreigners was .738.

Validity and suitability. First, I calculated the correlation between the monkeypanda-banana and the WoT scale to confirm their validity. If both scales are reflecting the holistic thinking validly, they should have a stable correlation with each other. However, the correlation between them in type $\mathrm{A}+\mathrm{B}+\mathrm{C}$ was -.045 , which means that there was no correlation at all between the two scales that should measure the same holistic thinking. This result indicates that at least one of the followings is true:

1. At least one of the holistic thinking scales in this study is not valid.

2. The theory of 'holistic thinking' itself is wrong. 
The first may be true because the reliability of the WoT scale was low and most scales did not satisfy the assumption of normality (I will explain it again in a moment.). Then how about the second? I think that the problem of research design alone cannot explain all of the negative correlation and the drastically low reliability.

The next is the external validity of the conservatism scale. In the questionnaire type $\mathrm{C}$, the correlation between conservatism and the self-reported political stances was .606. The average conservatism of Korean participants who voted for Park Geun-Hye in the 18th presidential election was $4.7(n=8, S D=0.67)$, and the average of those who voted for Moon Jae-In was $3.79(n=16, S D=0.98)$. The difference between the two groups was statistically significant at $\alpha=.05 ; t(22)=2.354, p=.028$. The correlation between Koreans' conservatism and their self-reported political stances was .423. The average conservatism of the foreigners who supported the Democrats was $2.85(n=22, S D=1.34)$, and the average of those who supported the Republicans was $4(n=3, S D=1.22)$. The difference between the two groups was not statistically significant; $t(23)=-1.407, p=.173$. The correlation between the foreigners' conservatism and their self-reported political stances was .703.

According to these results, the validity of the conservatism scale seems to be sufficiently high for predicting both Koreans' and foreigners' actual political choices. The two Korean groups that previously voted for different candidates showed a statistically significant difference in the conservatism scale, and this scale was highly correlated with the Koreans' self-reported political stances. The foreigners showed an even higher correlation with the self-reported stances, although the difference between the Democrat and the Republican was not significant.

The foresight credibility of the hindsight bias scale in the questionnaire type $\mathrm{C}$ was as follows. For all participants, the average foresight credibility of argument A was 4.24 $(S D=1.47)$, and one of argument B was $4.15(S D=1.52)$. This difference was not statistically significant $(p=.712)$. For Koreans, the average credibility of argument A was 4.65 $(S D=1.56)$, and one of argument B was $4.1(S D=1.52)$. For foreigners, the average of argument A was $3.83(S D=1.26)$, and one of argument B was $4.2(S D=1.54)$. The correlation between the dummy variable coded as 1 for foreigners and 0 for Koreans and the difference between the foresight credibility of argument A and one of argument B was -.185 ( $p=.100)$, and the correlation between the dummy variable and the sum of the foresight credibility was $-.225(p=.044)$. The significant correlation between the dummy variable and the sum means that Koreans are more likely to think that both arguments A and B are generally correct.

The monkey-panda-banana scale was not significantly correlated with the difference in the foresight credibility $(-.046)$ and with the sum of the foresight credibility $(.185$, $p=.101)$. The WoT scale was also not significantly correlated with the difference in the foresight credibility $(-.014)$ and with the sum of it $(-.100)$. However, the conservatism was significantly correlated with the difference in the foresight credibility $(.267, p=.017)$ and with the sum of it $(.310, p=.005)$. It means that conservatives tend to put their trust in argument A more than B and generally think that both arguments A and B are correct ${ }^{6}$.

Based on these results, I decided to maintain the assumption that people's credibil-

\footnotetext{
${ }^{6}$ This result is quite important because Nisbett argues that when encountering conflicting information, Asians are more likely to say 'both are correct' compared to Western people. However, this analysis indicates that such a phenomenon is due to conservatism rather than holistic thinking.
} 
ity of arguments A and B are all the same, despite some differences occurred by ethnic background and individual traits. However, there may be substantial measurement errors when measuring the influence of conservatism on hindsight bias because people's foresight judgments, which have to be the reference of the hindsight judgments, were found to be affected by conservatism. Nevertheless, I supposed that the impact of this problem on the results would be limited because the survey not only asks the credibility of both arguments but also presents the description that argument $\mathrm{A}$ is correct as well as one that argument $\mathrm{B}$ is correct. They will countervail the one-sided measurement errors.

The final issue was whether it is appropriate to classify the foreigners with various nationalities and lengths of residence into a single group. Therefore, I identified in the questionnaire type $\mathrm{A}+\mathrm{B}+\mathrm{C}$ whether the differences in monkey-panda-banana, the WoT, and conservatism exist based on the participants' nationality $]^{7}$ and the length of residence in Korea.

First, the score of the monkey-panda-banana scale of North Americans $(M=9.43$, $S D=2.69)$ and one of the foreigners other than North Americans $(M=8.85, S D=3.30)$ reported no statistically significant difference; $t(118)=1.045, p=.298$. The average WoT score of North Americans was $7.40(S D=2.62)$, and the average of the foreigners other than North Americans was $8.18(S D=2.26)$. This difference was not statistically significant; $t(118)=-1.754, p=.082$. The conservatism of North Americans $(M=3.47, S D=1.32)$ and one of the foreigners other than North Americans $(M=3.42, S D=1.22)$ showed no significant difference $(p=.831)$. The foreigners' length of residence was not significantly correlated with monkey-panda-banana (.064), the WoT $(-.060)$, and conservatism $(-.151, p=.351)$. These results imply that I do not have to consider the foreigners' nationality and the length of residence in the analysis. However, because it is possible to question that there may be some differences between North Americans and other foreigners, I decided to analyze North Americans and the foreigners other than North Americans separately.

\section{Main Analysis}

Normality. The Kolmogorov-Smirnov test showed that all major variables in this study were not normally distributed. The monkey-panda-banana (Lilliefors $p<.001$ ), the WoT (Lilliefors $p<.001$ ), the conservatism (Lilliefors $p=.006$ ), the hindsight bias (Lilliefors $p<.001$ ), and the negligence (Lilliefors $p<.001$ ) scales were all far from the normal distribution. Furthermore, the monkey-panda-banana and the WoT scales were found to be highly skewed; -1.334 and -0.555 , respectively, which means that most people generally agreed with the descriptions and got high scores. Although the sample size in this study is large enough to apply the central limit theorem, I will adopt nonparametric statistics for the rigorous analyses of the group differences. However, to help the readers' understanding of the results, I presented the averages of the variables as representative values so that the readers can intuitively compare the data.

Cultural difference in hindsight bias. Figure 1 displays the difference in hindsight bias between Koreans and foreigners in the questionnaire type A+B. Although I hypothesized that the hindsight bias of Koreans is higher than the foreigners,' the measured average was lower in Koreans $(M=0.5, n=80)$ rather than foreigners other than North

\footnotetext{
${ }^{7}$ As mentioned above, almost all foreign participants were either North Americans or Europeans.
} 
Americans $(M=1.09, n=44)$ and North Americans $(M=1.42, n=36)$. However, the KruskalWallis test showed that this difference was not statistically significant; $\mathrm{H}(2)=3.681, p=.159$. It means there was no difference in hindsight bias between Korean and foreign participants.

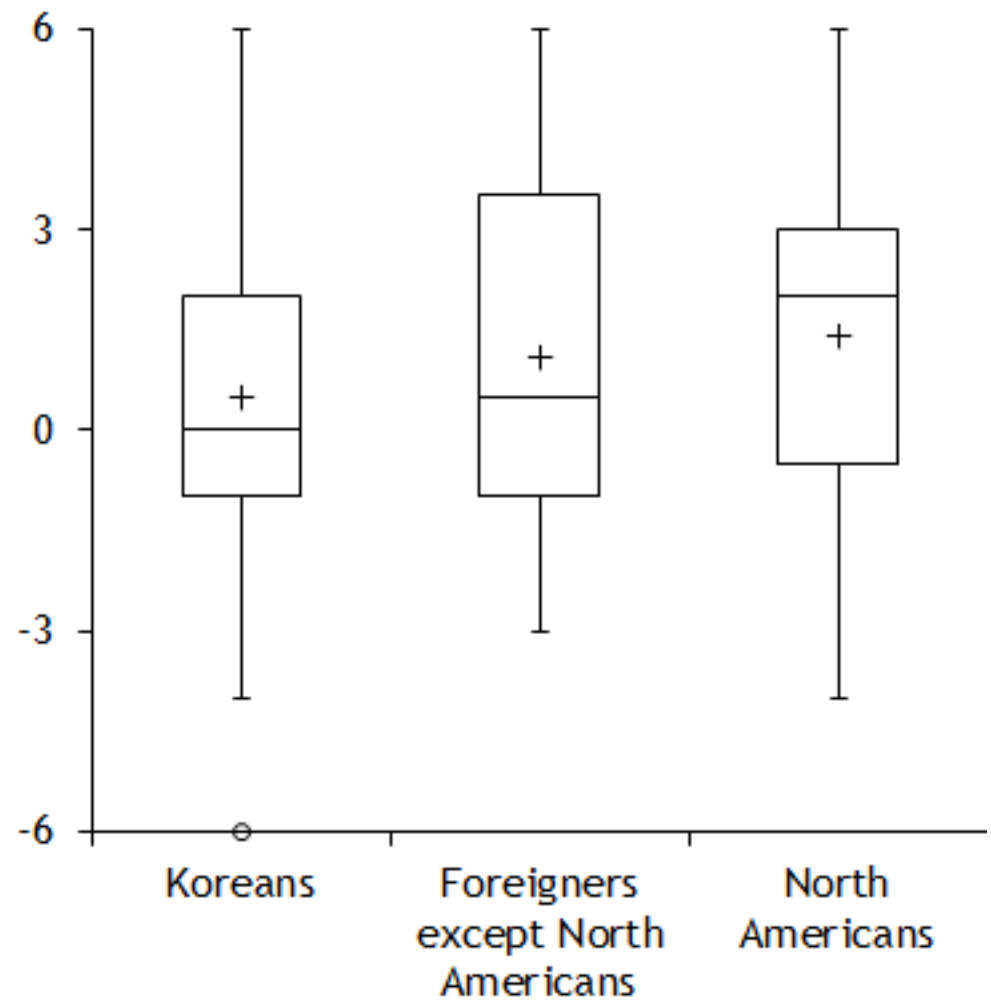

Figure 1. The difference in hindsight bias between Koreans and foreigners.

(Note: The crosshairs are the means and the circle is the outlier.)

Because the results were insignificant, I needed to check in more detail whether such results really support the null hypothesis or were simply due to the measurement insensitivity. I adopted the Bayes factor analysis suggested by Dienes (2014) for this. To set the possible domain of difference, I used the five nationality types mentioned above and calculated the biggest difference among them. Among the five groups, African/Arabian/Asian group reported the highest hindsight bias level $(M=1.6, n=5)$ whereas Eastern/Southern European and Latin American group reported the lowest $(M=0.46, n=13)$, and their difference was 1.14. Therefore, I assumed that this analysis could have the lowest bound of difference level of 0 and the highest bound of 1.14. Based on this assumption, the $B$ factor at the difference between Koreans and foreigners other than North Americans was $0.19(d=-0.59, F=2.03, S E=0.41)$ and the $B$ factor at the difference between Koreans and North Americans was $0.30(d=-0.92, F=2.03, S E=0.64)$. It seems that this analysis supports the null hypothesis rather than the alternative hypothesis, indicating that Koreans have no more hindsight bias than foreigners or Westerners.

In this analysis, the hindsight bias level of Koreans was rather numerically lower than the level of foreigner groups, and such results were opposite to the theory of cultural 
differences in hindsight bias. However, perhaps it is too hasty to conclude that Koreans' hindsight bias is no different from Westerners because I have to take account of some flaws or limitations in the design or procedure of this experiment. To illustrate why Koreans' hindsight bias was measured to be low, first I want to make sure that the two groups may have been in different conditions. Whereas most foreigners were travelers who were relaxed and had plenty of time, most Koreans were busy and could not fully concentrate on the surveys. Assuming that the probability that the participants respond to the manipulation in the survey is proportional to their concentration on the questionnaire, it may have been easier for foreigners to show hindsight bias. Indeed, the response rate of Koreans was lower than foreigners as well as they tended to answer faster than foreigners. Nevertheless, this result was clearly in direct opposition to Choi and Nisbett (2000)'s study which argued that Koreans have higher hindsight bias than Westerners. They claimed that as Koreans tend to see the complicated relationships compared to Westerners, they are easier to explain the unexpected results, so that they are not surprised at the unexpected results and more likely to say that "Things like that happen, too." Like their research, the strong inverse correlation between surprise and hindsight bias was also shown in this study (-.665).

However, the problem is that the self-reported surprise and the real surprise in people's cognitive process may be completely different. Using Pezzo (2003)'s sense-making theory once again, the reaction of surprise to the unexpected situation provokes the act of sensemaking, which consequently increases hindsight bias. In contrast, if the act of sense-making fails, the hindsight bias decreases. According to this theory, Koreans' strong hindsight bias in Choi and Nisbett (2000)'s study does not mean that they were not surprised in reality, but it is reasonable to think that they succeeded to rationalize themselves who had been surprised: They answered they were 'not surprised' because they found the plausible reason for rationalizing their surprise.

Cultural differences in holistic and analytic thinking. Figure 2 shows the difference in the monkey-panda-banana scale between Koreans and foreigners in the questionnaire type $\mathrm{A}+\mathrm{B}+\mathrm{C}$. The score of Koreans $(M=9.18, n=120)$ did not differ from foreigners other than North Americans $(M=8.85, n=62)$ and North Americans $(M=9.43, n=58)$. The Kruskal-Wallis test result was not statistically significant; $\mathrm{H}(2)=1.785, p=.410$.

The biggest difference in the monkey-panda-banana scale among the five nationality groups was 2.85, between Western/Central/Northern Europeans and Oceanians $(M=9.68$, $n=40)$ and Eastern/Southern Europeans and Latin Americans $(M=6.82, n=17)$. Therefore, the lowest bound was 0 and the highest was 2.85 . The $B$ factor at the difference in the monkey-panda-banana scale between Koreans and foreigners other than North Americans was $0.40(d=0.33, F=0.46, S E=0.48)$ and the $B$ factor between Koreans and North Americans was $0.10(d=-0.25, F=0.46, S E=0.36)$. Based on the $B$ factors, the Koreans' monkey-panda-banana score does not seem to be different from the foreigners' scores.

In addition, I think it is more important to consider that in this study, most participants preferred the classification based on relationship rather than categorization, regardless of the nationalities or ethnic background. Although we cannot say with only this study that the cultural differences in the way of thinking do not exist, the crucial fact is that not only Koreans but also Westerners strongly preferred the relationship-based answers. Did the Westerners on the Korean TV show who tended to automatically connect monkey to panda originate from the different planet from those in my study? I could not find any evidence 


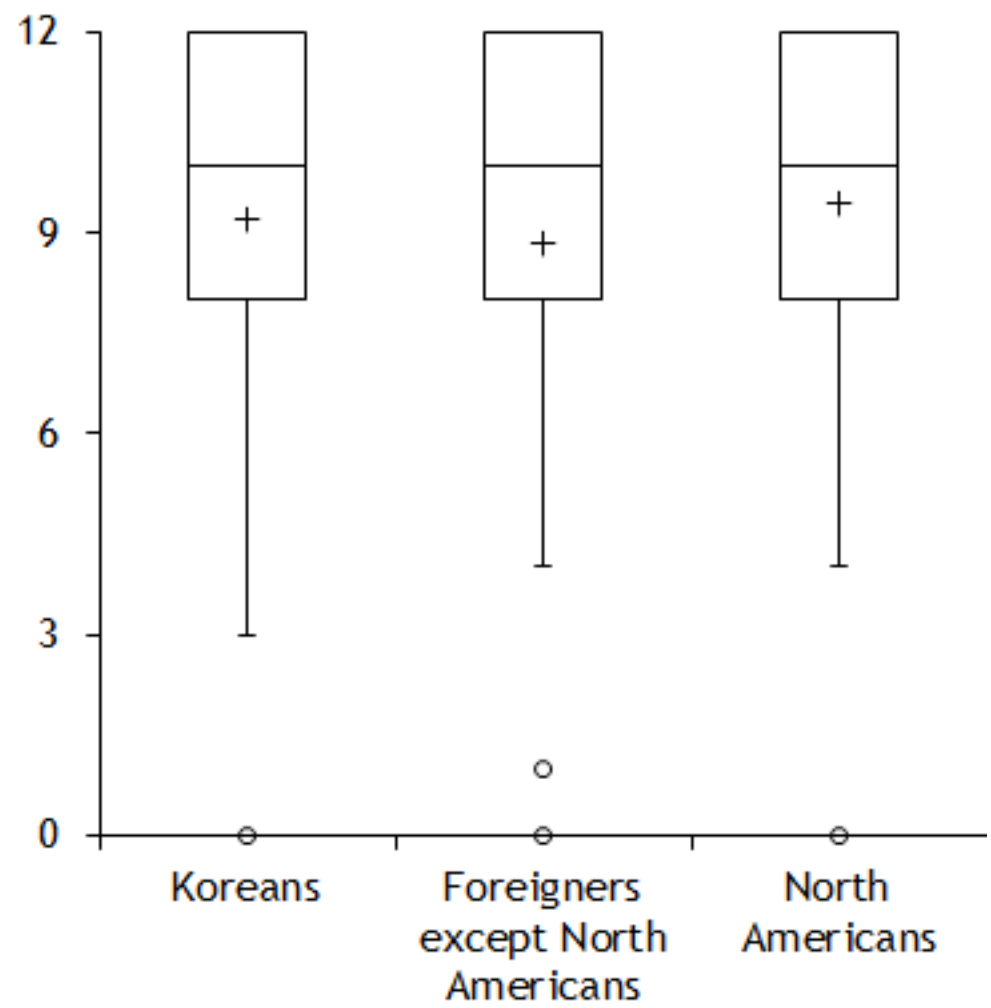

Figure 2. The difference in the monkey-panda-banana scale between Koreans and foreigners.

that there are two kinds of Westerners.

Next, Figure 3 displays the difference in the WoT scale between Koreans and foreigners in type $\mathrm{A}+\mathrm{B}+\mathrm{C}$. Koreans $(M=8.23, n=120)$ and foreigners other than North Americans $(M=8.18, n=62)$ reported higher scores than North Americans $(M=7.40, n=58)$, but this difference was not statistically significant in the Kruskal-Wallis test; $\mathrm{H}(2)=4.154, p=.125$.

The biggest difference in the WoT scale was 1.13 between Eastern/Southern Europeans and Latin Americans $(M=8.53, n=17)$ and Canadians/Americans $(M=7.40, n=40)$. Therefore, the lowest bound was 0 and the highest was 1.13. The $B$ factor at the difference in the WoT scale between Koreans and foreigners other than North Americans was 0.26 $(d=0.06, F=2.58, S E=0.03)$ and the $B$ factor between Koreans and North Americans was $2.77(d=0.84, F=2.58, S E=0.52)$.

It seems that $B$ factor at this scale supports the alternative hypothesis more than the null hypothesis, although the difference between Koreans and foreigners other than North Americans supported the null hypothesis more. Indeed, there was a numerically observable tendency that North Americans preferred analytic thinking more than other groups, albeit statistically insignificant. However, considering that the foreigners other than North Americans were also mostly Westerners, it is unlikely to think that this difference is the 'East-West' difference. Furthermore, Koreans, foreigners other than North Americans, and North Americans tended to answer inconsistently for each item. Of the four items in this scale, the first two items showed that Koreans $(M=2.13)$ answered more holistically 


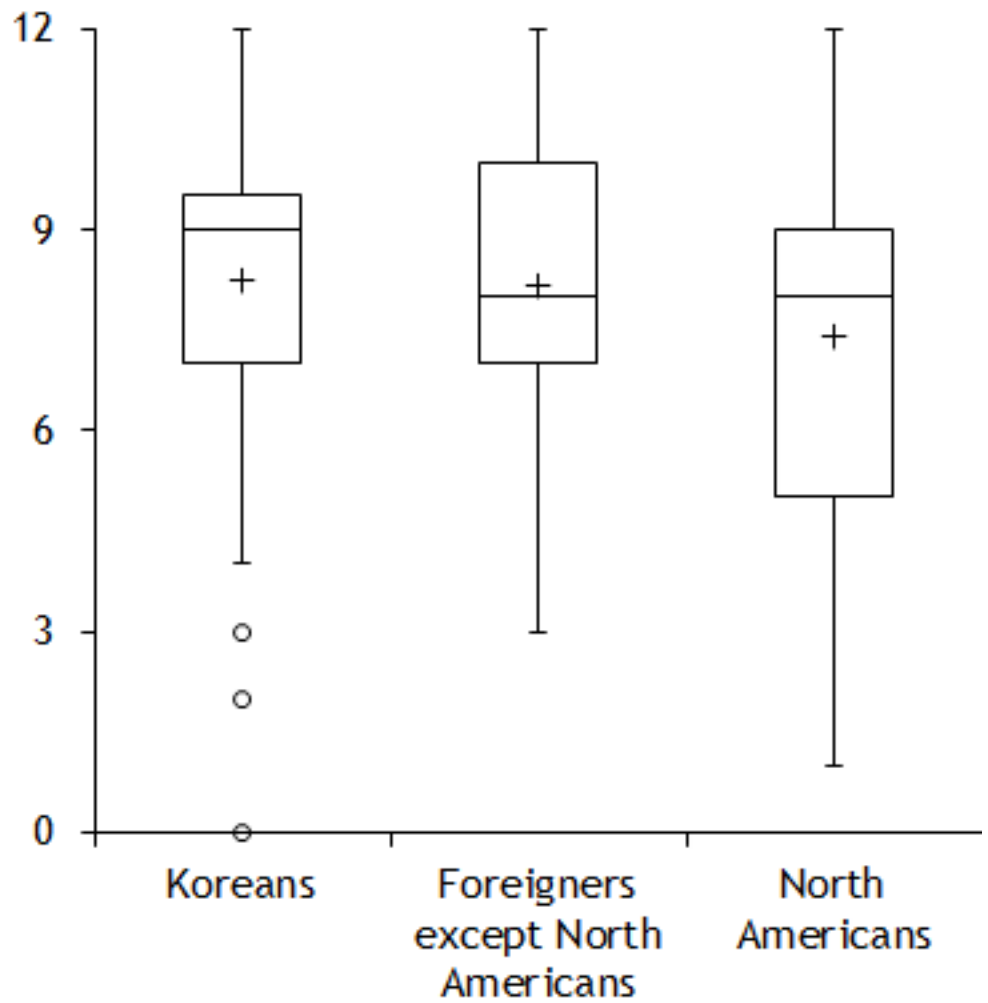

Figure 3. The difference in the way-of-thinking scale between Koreans and foreigners.

than foreigners other than North Americans $(M=1.93)$ and North Americans $(M=1.60)$. However, the third question was different. Compared to Koreans $(M=2.07)$, foreigners other than North Americans $(M=2.40)$ and North Americans $(M=2.59)$ reported higher scores.

I can say that the result of the third item is predictable to some degree, unless it is also a sort of hindsight bias. The third item apparently described the Mother Nature, but the essential and analytic description was rather close to Eastern philosophy, whereas the description that drew the analogy between nature and a symphony is more familiar to Westerners rather than Easterners. It indicates that the holistic-analytic thinking is not the stable and solid cognitive process but rather the inconsistent one which is easily affected by cultural background or contexts. The extremely low reliability of this scale also supports this. However, the last item that described science, which does not seem to be influenced by cultural background, also showed some difference; Koreans $(M=1.92)$ and foreigners other than North Americans $(M=1.92)$ showed higher scores than North Americans $(M=1.60)$, which indicates that the concept of holistic thinking itself may not be wrong, even though the difference seems much smaller than Nisbett has argued.

Cultural difference in conservatism. Figure 4 shows the difference in the conservatism between Koreans and foreigners in the questionnaire type $\mathrm{A}+\mathrm{B}+\mathrm{C}$. Koreans $(M=3.91, n=120)$ reported higher scores in conservatism than foreigners other than North Americans $(M=3.42, n=62)$ and North Americans $(M=3.47, n=58)$. The Kruskal-Wallis test showed that this difference is statistically significant; $\mathrm{H}(2)=10.419, p=.005$. Besides, 
the Dunn-Bonferroni post-hoc test reported that there are differences between Koreans and foreigners other than North Americans $\left(p_{a d j}=.016\right)$ and between Koreans and North Americans $\left(p_{a d j}=.040\right)$.

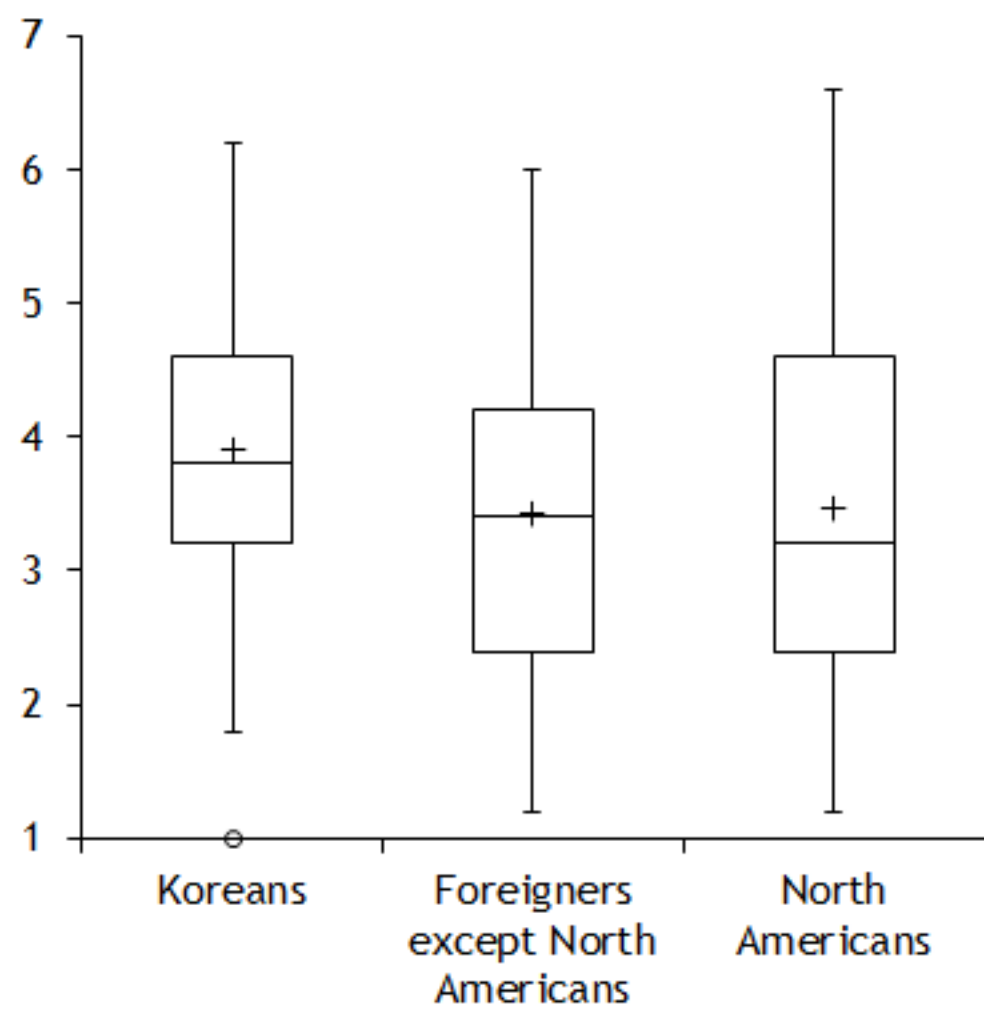

Figure 4. The difference in the conservatism between Koreans and foreigners.

In this scale, all items except the first item (general description of conservative identity) reported that the Korean participants' score was significantly higher than the foreigners. I interpreted it as follows: Koreans who do not report themselves as conservative actually have more conservative traits than foreigners, whereas the foreigners who think that they are conservative indeed disagree with the sentences that most Koreans accept. This implies two pivotal possibilities. First, the criteria of conservatism may not be absolute or universal. People in different cultures have different degrees of conservatism from each other, and those who have been conservative in some cultural area may not be conservative any more in another cultures, and vice versa. Second, self-reporting may not be the appropriate method to measure conservatism. Lee, Choi, and Hur (2012) reported that the Koreans' self-reported conservatism did not completely match the actual political stances.

Meanwhile, the item with the largest difference was the third one (discriminatory attitude against women). Koreans $(M=4.07)$ reported higher scores in this item compared to foreigners other than North Americans $(M=3.21)$ and North Americans $(M=3.36)$, indicating that the androcentric views and authoritarianism are still prevalent among the majority of Koreans. 
What boosts hindsight bias? The analyses so far demonstrated that there is no cultural difference in the monkey-panda-banana scale, the difference in the WoT scale is unclear and insignificant, and Koreans have higher hindsight bias than foreigners. Then, which factor(s) among them would affect hindsight bias? The existing 'holistic thinking' theory, conservatism, or both? To identify this, I performed a multiple regression analysis in the questionnaire type $\mathrm{A}+\mathrm{B}$ with the monkey-panda-banana, the WoT, and the conservatism scales as independent variables and the hindsight bias scale as a dependent variable.

In this regression model, the variance inflation factors in the analysis were close to 1 in all variables, and the plots of residuals showed no distributions indicating that the basic assumptions of regression model were violated. This model reported the low coefficient of determination; $\mathrm{F}(3,156)=1.857, R_{a d j}^{2}=.016$. The result showed that the monkey-pandabanana scale did not affect on hindsight bias $(\beta=-.016, p=.840)$ and so did the WoT $(\beta=-.042, p=.595)$. However, conservatism was found to be a factor that increases hindsight bias $(\beta=.179, p=.024)$.

Because I hypothesized that holistic thinking would raise hindsight bias because of the impact of field dependence, such a result that the regression coefficients were rather negative was quite a surprise to me. Because it is known that the field dependence increases hindsight bias, I have to conclude that the scales in this study could not measure people's field dependence appropriately. Then, was it a problem of my research design, the theory of holistic thinking, or both?

Meanwhile, although the influence of conservatism on hindsight bias was significant, I think it is hasty to conclude that conservatives tend to have more hindsight bias than others. The slight flaw in the research design may have increased the measurement error of hindsight bias: There is a possibility that the foresight answers to the arguments A and B may have been affected by hindsight bias. For example, we cannot say that the conservatives who answered that they thought argument A was correct always have hindsight bias because they actually tend to believe argument A is correct more than B. I think this phenomenon had a negative effect on this study and finally contributed to the low statistical power: The post-hoc statistical power of the regression analysis with the conservatism as an independent factor and the hindsight bias as a dependent factor was only .646 $\left(R^{2}=.032, n=160\right)$.

By the way, the correlation of Koreans' conservatism with hindsight bias was .163, and one of foreigners' conservatism with hindsight bias was .232. The regression analysis also showed that the impact of conservatism on hindsight bias increases when the influence of the dummy variable that indicates nationality is controlled $(\beta=.201, p=.011)$. However, I do not suppose that it means Koreans' hindsight bias is actually less affected by conservatism. As mentioned above, most Koreans failed to concentrate on the survey, probably decreasing the effect of the manipulations in this study.

Mediating effect on hindsight bias. Positing that the regression analysis above was accurate, I intend to verify my hypothesis that negligence is mediating the relationship between conservatism and hindsight bias. The regression model was established in questionnaire type $\mathrm{A}+\mathrm{B}$, investigating the relationship among the conservatism, negligence, and hindsight bias. In all regression analyses in this part, the variance inflation factors in the analyses were close to 1 in all variables, and the plots of residuals showed no distributions indicating that the assumptions were violated. First, conservatism strongly affected negligence $(\beta=.341, p<.001)$, but there was no relationship between negligence and hindsight 
bias $(\beta=.016, p=.845)$. It means that conservatives tend to think carelessly and are likely to be confident in their intuitive judgments, but this tendency does not increase hindsight bias. As the causal relationship between the negligence and the hindsight bias did not appear to be existent, I did not test the mediating effect of negligence.

This result indicates either the negligence scale could not precisely measure 'the catalyst for sense-making' that boosts hindsight bias, or my theory was incorrect from the beginning. If the former is true, it may also be the problem of self-reporting; As most participants could not understand not only the contents of the questionnaire but also the cognitive process of themselves, their reported answers would be derailed from the real tendencies. If the latter is true, I have to consider the different model that connects the conservatives' dogmatism, rigidity, and conscientiousness with the specific cognitive process.

I also have to consider whether the relationship between conservatism and negligence was due to the response bias such as acquiescence or carryover effect, as they are both seven-point Likert scales and the negligence scale is measured right after the conservatism. However, there is also a difference that cannot be explained by acquiescence: The correlation between conservatism and negligence was higher in foreigners (.491) than Koreans (.152). Although I could not clarify why the foreigners reported higher correlation, it is unnatural to think that the foreigners are likely to acquiesce in all sentences more than Koreans. Furthermore, even if the result supported the hypothesis, I would have to be careful to accept the result as it is. As well as the problem of self-reporting, the negligence scale itself has a critical validity problem, which I will talk about in a moment. 


\section{The Leftovers}

I think the results of this study are sufficient to seriously impact the existing theories of the East-West cultural differences in cognitive mechanisms. If such theories were true, the Koreans' scores of the monkey-panda-banana scale and the WoT scale should be higher than the foreigners' scores, and these phenomena should affect hindsight bias. None of them was proven to be right in this study, including main analyses and minor sub-analyses.

This results indicate that the cross-cultural theory of cognitive traits so-called 'holistic and analytic thinking' may not reflect the reality, but rather can be an artificial product made from the addition of the most plausible explanation of the alleged study results. Indeed, many studies in cultural psychology are being done like this; If someone finds a 'difference,' they usually do not thoroughly perform further verification of the causal relationship or consider the external variables that might affect the study. Instead, they usually prefer the confirmatory explanations of their results; The fatal problem is that such explanations are also possible in the opposite result. Therefore, it may be nothing but hindsight bias by itself.

However, someone would also argue that the negative results in this research might be fully explained by the problems of the experimental design or scale validity in the study. This study was not conducted perfectly and contains many intrinsic and extrinsic problems. The first problem is that as I mentioned above, most Koreans did not actively answer the survey compared to foreigners. This tendency caused a great deal of noise, especially when measuring hindsight bias: It was tough to demand surprise and sense-making to Koreans who were usually busy and answered briefly.

This problem may even distort the sampling process. In this study, I printed three to four pages of a questionnaire in two sheets, which were very cramped, and the lengthy contents may have caused the significant cognitive overload to the respondents. If so, those who do not like to learn about new information and solve problems with rational thinking, i.e., those whose needs for cognition are low, would likely to reject the survey, so that the final respondents may have had the higher needs for cognition compared to ordinary people. Furthermore, it is known that people with high needs for cognition are open to new thoughts and values (Fleischhauer et al. 2010) and less influenced by stereotypes or biases (Leary \& Hoyle, 2009), implying that the Korean participants in this study might have had lower conservatism and hindsight bias than ordinary Koreans.

To make the matter worse, the foreign participants in this study may also have had lower conservatism than the average foreigners. The participants in this study were not just foreigners but the foreigners who were traveling abroad or living in a different culture. Then, do such foreigner group have different cognitive traits from ordinary foreigners or Westerners? I thought that it may be. Openness to experience is a factor of the Big Five personality, which includes aesthetic sensibility, preference for diversity, and intellectual curiosity. By the way, McCrae (1996) suggests that this trait is negatively related to conservatism. Because people with higher openness to experience are usually less ethnocentric, more likely to accept things from different cultures (Sibley \& Duckitt, 2008), and always eager for new experiences, we can expect that they would likely to travel overseas or live in different cultural areas more than others. That is to say, foreigners that I met in Korea may have higher openness to experience and lower conservatism compared to other foreigners. 
There are lots of clues indicating that the Korean and foreign participants in this study did not fulfill the representativeness of the targeted groups. The issue is that the respondents in this study were mostly young people (20s or 30s), and the distribution of their political choices was disproportionate compared to the ordinary people's distribution. First, the average age of Koreans was 27.8, and one of the foreigners was 29.6. Although I cannot analyze the older groups' data here, they were similarly young, and this difference will not impact seriously on the experiment. Meanwhile, the Korean respondents reported that 8 of them voted for Park and 16 of them voted for Moon. It differs greatly from the actual distribution 8 , but it also seems to be similar to the actual percentage of young Koreans (The 18th Presidential Election Exit Poll, 2012, December 19). In contrast, 22 of foreigners answered that they support the Democratic Party whereas only three of them supported the Republican Party: Although supporting and voting may be different because the latter includes floats or swing voters, it seems true that the representativeness was more distorted in the foreigner group than the Korean group.

If the measurement of conservatism was distorted more in the foreigner group than Korean group, i.e., if the foreigner sample in this study had the lower average of conservatism than the ordinary foreigners, the result of this study that Koreans were more conservative than foreigners can also be called into question. Although I gauge the result would not be changed even if this problem got resolved, at least it may be true that the difference was over-measured compared to the actual one, especially because the problem also exists in the conservatism scale itself as well as the sampling process. The problem of the conservatism scale was already mentioned above: Because the five questions were all the same direction and there were no reverse-scored questions, the response bias such as acquiescence may have occurred. Although it was an inevitable decision in some degree because I had to minimize the length of the questionnaire and secure the reliability with the brief item set, this scale would likely to make the conservatism of those who agree with all sentences overestimated compared to others.

This problem may be crucial. As I mentioned earlier, Nisbett argues that Easterners are likely to say that 'both are right' whereas Westerners tend to choose and accept only one of the contradictory information. What if Koreans' high conservatism was due to the so-called 'bothsidesism' rather than their actual conservative tendencies? Then Nisbett can maintain his theory because it unintentionally measured holistic thinking rather than conservatism. To conclude, that is unlikely to happen. The validity check in this study showed that the measured conservatism had decent correlations with the actual political choices. Furthermore, the questions of this scale adequately contain exclusiveness and dogmatism, which are considered as the major characteristics of conservatism, so that the real 'bothsidesists' would not (at least strongly) agree with such one-sided sentences. In particular, the fourth and fifth questions of conservatism ask traits to exclude or attack those who are different from one's thoughts or those who disagree with oneself, so the majority of bothsidesists would disagree with them. Then what if it was just acquiescence, i.e., the respondents tended to agree with all the sentences without particular opinions? Then it would generate noises and hinder the precise measurement of conservatism, which may consequentially have affected the validity of the scale.

${ }^{8}$ President Park won in this election. 
Were there any problems with other scales? The monkey-panda-banana scale was not normally distributed and had too many extreme values, as I mentioned above?. Furthermore, I cannot say that my scale was entirely objective and unbiased; For example, 'sugar' may refer to the generic term of all saccharides rather than only the processed goods made from sugar cane. Therefore, foreigners might have felt awkward to classify honey and sugar with the same category because they may have thought that sugar includes honey. However, in Korea, sugar refers only to the powder made from sugar cane, so that they would not be confused classifying them.

The hindsight bias scale also had a significant problem. I assumed that the people's foresight credibility of arguments A and B should be all the same and demonstrated that the 'average' foresight credibility of people was not significantly different. However, I ignored not only some proven group differences but also standard deviations or within-group variances, which may be even more crucial than the average. If the standard deviation of the foresight credibility is too high, it will become the measurement error of this scale by itself. Thus, now I think that it was the primary reason why the effect size of the relationship between the conservatism and the hindsight bias seemed inadequate.

The negligence scale was a minor factor in this study, but it was far from well-made. Because the last two items of this scale had almost no internal consistency, I had to remove them, which means the experiment did not go as well as I expected. As I had to remove two of four items, there was a question mark over the validity of this scale.

Those I discussed so far are the problems in this study that I could discover. They are all the catalysts that may have affected the results or at least amplified the measurement errors in the analysis. However, I believe that the data in this study sufficiently proves that even if these problems could all be cleared away, it would be unlikely to think that the alleged 'big cultural difference in the way of thinking' will appear. Even though this study is in some ways flawed, it is impossible to explain why most Western people connected coke with burger rather than water: I have no choice but to conclude that the difference between holistic and analytic classification does not exist in the ordinary situations.

Assuming that the result of this study was right or at least acceptable, there is only one way to maintain this theory: As I said above that foreigners in Korea might be less conservative than ordinary foreigners, they might also be more holistic than ordinary foreigners. In this case, the two possibilities can be considered. First, after the foreigners start to live in Korea, they may have been exposed to Korean culture in their life and gradually have become holistic. At the end of the study, I can say that such possibility is rejected because the foreigners' length of residence did not correlate with the monkeypanda-banana and WoT scales.

The remaining possibility is that they were already familiar with Korean culture before coming to Korea. Where could they know Korean traditions and values? The participants in this study all looked like Westerners, and I did not contact those who look like Asians or mixed Asians. It seems evident that at least the majority of them could not see Korean or Oriental culture in their family background. Of course, they could know Korean culture indirectly through their friends, media, or the Internet, and they might have had special interests in or goodwill towards Korea. However, it is weird to think that the peripheral

\footnotetext{
${ }^{9}$ The dummy or filter items in between the real ones may have reduced this problem, but the questionnaire would be too long if I did so.
} 
influences of Korean culture outside the family are absolute enough to change their cognitive traits. But do not be disappointed because there is still a perfect possibility: As soon as the foreigners who were initially analytic came into Korea, they smelled magical garlic breath from the airport and suddenly became holistic!

Sorry for the lengthy writing, and here is my last discussion. I think research in cultural psychology is so difficult. I mean, to compare the two or more groups accurately, we have to strictly and thoroughly control all the conditions other than the conditions that we intend to manipulate, or at least minimize the noises that may come from unexpected conditions or events. In cultural psychology, it is incredibly tough, because compared to other academic areas, the studies in cultural psychology must be conducted under considerably different conditions between groups: different places, different times, different tools, different languages, and different and irrelevant cultural backgrounds. However, that is why the cross-cultural research should be more rigorous. If someones establish a hypothesis and find a significant difference in their study, is there a way to determine whether it resulted from intended experimental manipulation or from unexpected noises? Have cultural psychologists made adequate efforts to minimize noises from different collective traits or precisely conducted analyses that confirm experimental results and negate the effects of extraneous variables?

At the end of his book, Nisbett wrote: "In almost all of the studies we have conducted, Asians and Westerners have shown differences in mindset, and they have also been huge." (Nisbett, 2003) In only about a decade, the once huge difference has become so small that it cannot be statistically attested. I think I have conveyed in a rational manner throughout this study how far this argument is from the reality. Nevertheless, this study might be just an assertion, and there may still be some people who do not believe this. However, at least it is clear that this cross-cultural research of cognitive tendency has the typical problems of the contemporary studies in cultural psychology, represented by the dichotomous distinction between Easterners and Westerners, the naive and unsystematic methodology, and the imprecise statistical analyses. In addition, I think it is time for us to seriously consider whether adding contrived interpretations on one-sided study results and publishing them without least evidence of causality is nothing more than hindsight bias. 


\section{References}

The 18th presidential election exit poll. (2012, December 19). Retrieved from https://news.sbs . co.kr/news/endPage.do?news_id=N1001540823

Choi, I., Koo, M., \& Choi, J. A. (2007). Individual differences in analytic versus holistic thinking. Personality and Social Psychology Bulletin, 33(5), 691-705.

Choi, I., \& Nisbett, R. E. (1998). Situational salience and cultural differences in the correspondence bias and actor-observer bias. Personality and Social Psychology Bulletin, 24(9), 949-960.

Choi, I., \& Nisbett, R. E. (2000). Cultural psychology of surprise: holistic theories and recognition of contradiction. Journal of Personality and Social Psychology, 79(6), 890.

Dienes, Z. (2014). Using bayes to get the most out of non-significant results. Frontiers in psychology, $5,781$.

Fischhoff, B. (2007). An early history of hindsight research. Social cognition, 25(1), 10-13.

Fleischhauer, M., Enge, S., Brocke, B., Ullrich, J., Strobel, A., \& Strobel, A. (2010). Same or different? clarifying the relationship of need for cognition to personality and intelligence. Personality and Social Psychology Bulletin, 36(1), 82-96.

Gerber, A. S., Huber, G. A., Doherty, D., Dowling, C. M., \& Ha, S. E. (2010). Personality and political attitudes: Relationships across issue domains and political contexts. American Political Science Review, 104(01), 111-133.

Gong, S. (1996). Interpersonal perception, contextual attribution: The cross-cultural approach (Unpublished master's thesis). Chonnam National University.

Heine, S. J., \& Lehman, D. R. (1996). Hindsight bias: A cross-cultural analysis. The Japanese Journal of Experimental Social Psychology, 35, 317-323.

Ji, L. J., Zhang, Z., \& Nisbett, R. E. (2004). Is it culture or is it language? examination of language effects in cross-cultural research on categorization. Journal of Personality and Social Psychology, 87(1), 57.

Jost, J. T., Glaser, J., Kruglanski, A. W., \& Sulloway, F. J. (2003). Political conservatism as motivated social cognition. Psychological Bulletin, 129(3), 339.

Kanai, R., Feilden, T., Firth, C., \& Rees, G. (2011). Political orientations are correlated with brain structure in young adults. Current Biology, 21(8), 677-680.

Krull, D. S., Loy, M. H.-M., Lin, J., Wang, C.-F., Chen, S., \& Zhao, X. (1999). The fundamental fundamental attribution error: Correspondence bias in individualist and collectivist cultures. Personality and Social Psychology Bulletin, 25(10), 1208-1219.

Leary, M. R., \& Hoyle, R. H. (2009). Handbook of individual differences in social behavior. Guilford Press.

Lee, J., Choi, S., \& Hur, T. (2012). The implicit theories of human and society in political preferences. The Korean Journal of Social and Personality Psychology, 26 (2), 69-86.

McCrae, R. R. (1996). Social consequences of experiential openness. Psychological Bulletin, 120(3), 323.

Musch, J. (2003). Personality differences in hindsight bias. Memory, 11(4-5), 473-489.

Nisbett, R. E. (2003). The geography of thought. Free Press.

Nisbett, R. E., Peng, K., Choi, I., \& Norenzayan, A. (2001). Culture and systems of thought: holistic versus analytic cognition. Psychological Review, 108(2), 291.

Pezzo, M. (2003). Surprise, defence, or making sense: What removes hindsight bias? Personality and Social Psychology Bulletin, 11(4-5), 421-441.

Pohl, R. F., Bender, M., \& Lachmann, G. (2002). Hindsight bias around the world. Experimental Psychology, 49(4), 270-282.

Sibley, C. G., \& Duckitt, J. (2008). Personality and prejudice: A meta-analysis and theoretical review. Personality and Social Psychology Review, 12(3), 248-279.

Tetlock, P. E. (1983). Cognitive style and political ideology. Journal of Personality and Social Psychology, 45(1), 118. 